Acta Poetica $33 \bullet 1$

ENERO-JUNIO

$2012(19-47)$

\title{
Maniobras estratégicas: combinando lo razonable y lo efectivo en el discurso argumentativo
}

\author{
Frans H. van Eemeren
}

Después de explicar su concepción de la argumentación como tema para teorizar, Frans H. van Eemeren presenta un esbozo del programa de investigación del acercamiento pragma-dialéctico a la argumentación, introduciendo los principales componentes de la teoría de la argumentación que resultan del desarrollo de este programa. Posteriormente se centra en el problema fundamental que confrontan los teóricos de la argumentación al buscar distinguir entre los movimientos argumentativos razonables y los falaces. Al respecto, presenta el tratamiento pragma-dialéctico de las falacias como una alternativa constructiva a las severas críticas dirigidas al tratamiento estándar lógico. En la perspectiva pragma-dialéctica, las falacias se consideran violaciones de las reglas para conducir una discusión crítica orientada a resolver una diferencia de opinión de fondo, lo que está en juego en el discurso argumentativo. Van Eemeren introduce la noción de maniobra estratégica para explicar el poder persuasivo de las falacias, a pesar de tratarse de argumentaciones que van en contra de lo razonable. La noción de maniobra estratégica captura el predicamento inherente a todo movimiento argumentativo de reconciliar, a la vez, la búsqueda de resultados efectivos con la necesidad de respetar parámetros razonables. Las falacias son descarrilamientos de las maniobras estratégicas que violan una o más reglas de la discusión crítica. A través de la distinción entre un argumento de autoridad razonable y el argumentum ad verecundiam (que corresponde con una falacia) van Eemeren concluye su ensayo explicando, con este ejemplo, por qué los descarrilamientos de las maniobras estratégicas pueden, en la práctica, pasar tan fácilmente inadvertidos, lo que ayuda a que las falacias no sean detectadas.

PALABRAS ClaVE: pragma-dialéctica, discusión crítica, falacia, maniobra estratégica, argumentum ad verecundiam. 
After explaining his view on argumentation as a subject matter for theorizing, Frans H. van Eemeren sketches the research program of the pragma-dialectical approach to argumentation and introduces the main components of the theory of argumentation resulting from carrying out this program. Next he concentrates on the fundamental problem argumentation theorists are confronted with of distinguishing between sound and fallacious argumentative moves. He presents the pragma-dialectical treatment of the fallacies as a constructive alternative to the severely criticized Logical Standard Treatment. In the pragmadialectical approach fallacies are treated as violations of rules for conducting a critical discussion that is aimed at resolving a difference of opinion which is at issue in argumentative discourse on the merits. To tackle the problem of the persuasiveness that fallacies, in spite of their unreasonableness, may have, van Eemeren introduces the notion of strategic maneuvering, which captures the predicament inherent in every argumentative move of having to reconcile aiming for effectiveness and maintaining reasonableness. Fallacies are derailments of strategic maneuvering in which one or more of the rules for critical discussion have been violated. Using the distinction between a sound argument from authority and the fallacy of argumentum ad verecundiam as a case in point, van Eemeren concludes his essay by discussing the reasons why derailments of strategic manoeuvring can in practice go so easily unnoticed, so that fallacies are not detected.

KeYwORDS: Pragma-dialectics, critical discussion, fallacy, strategic maneuvering, argumentum ad verecundiam.

Fecha de recepción: 9 de agosto del 2011

Fecha de aceptación: 29 de marzo 2012 
Frans H. van Eemeren

Universidad de Amsterdam, Países Bajos

\section{Maniobras estratégicas: combinando lo razonable y lo efectivo en el discurso argumentativo}

[Traducción del inglés de Luisa Puig]

\section{La argumentación como tema para teorizar ${ }^{1}$}

El estudio de la argumentación prospera. Este es un hecho notable y afortunado, ya que durante un largo periodo de abandono parecía haber desaparecido para siempre del escenario académico. Tras su brillante comienzo en la Antigüedad, realzado con las obras clásicas de Aristóteles, tras una alternancia de ascensos y descensos en los siguientes milenios, en el periodo post-renacentista se inicia su gradual decadencia. $\mathrm{La}$ revitalización tuvo lugar solo después de que Toulmin y Perelman publicaran, en el mismo año (1958), sus trascendentales obras The Uses of Argument y La nouvelle rhétorique (en coautoría con Olbrechts-Tyteca y traducida al inglés en 1969). ${ }^{2}$ El modelo de la argumentación de Toulmin y el inventario de técnicas de la argumentación de Perelman inspiraron a muchos especialistas para retomar seriamente el estudio de la argumentación de muy diversas formas. Hoy en día se encuentran acercamientos lógicos (formales así como informales) bien establecidos, así como comunicativos, lingüísticos, sociales, psicológicos, jurídicos y de

${ }^{1}$ Este trabajo se basa en las ideas centrales de mi conferencia en el Congreso de Argumentación en Galati, Rumanía, el 14 de mayo de 2010.

${ }^{2} \mathrm{Y}$ al español, en la editorial Gredos en 1989 [nota de la traductora]. 
otros tipos. Es posible encontrar las huellas de la influencia clásica y neoclásica de las teorías de la argumentación antes mencionadas en la mayoría de estos acercamientos (van Eemeren et al., Fundamentals of Argumentation Theory).

Desde la Antigüedad es una evidencia reconocida que la argumentación surge como respuesta o en anticipación a una diferencia de opinión, real o meramente imaginaria. Cuando las personas argumentan a favor de su caso, están defendiendo una opinión o un "punto de vista" que asumen como no compartido por el destinatario o por un tercero con el que se asocia el destinatario - de lo contrario, la argumentación no tendría razón de ser (van Eemeren y Grootendorst, Speech Acts in Argumentative Discussions, 39-46). La necesidad de argumentar, los requisitos de una justificación y la estructura de la argumentación están todos adaptados a un contexto en el que surgen dudas, oposición, objeciones y contraobjeciones.

Al teorizar sobre la argumentación es vital comprender que los puntos de vista en cuestión en una diferencia de opinión pueden tratar sobre cualquier tema y, a la vez, ser descriptivos así como evaluativos o prácticos. En un discurso argumentativo, un juicio evaluativo, como "La película Infamous es brillante" o la orden práctica de llevar a cabo alguna acción, por ejemplo, "Deberías acompañarme a la junta", pueden ser objeto de una discusión, de la misma manera que una afirmación descriptiva sobre un estado real de cosas como "Amsterdam es mucho más grande que Rotterdam". Los puntos de vista de todos estos tipos y la argumentación que los sustenta, pueden encontrarse en cualquier terreno, desde el círculo familiar y la escuela hasta el derecho y la arena política, y es imperativo que el estudio de la argumentación se ocupe de toda la gama de las prácticas argumentativas.

Ciertos filósofos sostienen el parti pris de que las afirmaciones normativas, como son los puntos de vista evaluativos y prescriptivos -a los que tradicionalmente se llama "prácticos"-, nunca pueden ser sometidas a una discusión racional. Por su parte, algunos teóricos de la argumentación que la consideran como "un asunto fundamentalmente epistémico" alimentan este prejuicio, asignando un estatus superior a las afirmaciones descriptivas, las cuales se considera que cumplen un papel especial en el proceso de búsqueda y preservación de la verdad al "llevar a los 
razonadores de las verdades reconocidas o de las creencias justificadas, a verdades no reconocidas de antemano o a creencias no justificadas de otra forma" (Biro y Siegel, "Normativity, Argumentation and an Epipstemic Theory of Fallacies", 99). Otros especialistas de la argumentación, al tomar un enfoque retórico, favorecen el otro extremo al considerar que la argumentación retórica trata únicamente de afirmaciones relativas a la acción y, por lo tanto, fomentan la exclusión de los puntos de vista descriptivos y evaluativos (Kock, "The Domain of Rhetorical Argumentation").

La consecuencia principal de excluir del estudio de la argumentación, de manera cuasi-positivista, los puntos de vista evaluativos y prácticos es que los juicios de valor y las elecciones para la acción se relegan enteramente a preferencias subjetivas e intereses personales. Al contrario de estos filósofos con una perspectiva tan "exclusionista", John Stuart Mill — quién mostró gran interés por la discusión de los puntos de vista éticos, políticos y religiosos - junto con otros filósofos "inclusionistas" afines, considera que cualquier tema puede ser objeto de una discusión racional argumentativa (Finocchiaro, "Mill's On Liberty and Argumentation Theory"). Estoy absolutamente de acuerdo con Mill y con los otros representantes de esta tradición analítica, y no encuentro justificación alguna en defender posiciones que impliquen una jerarquía de valores o un principio de acción que a priori sean inapropiados para este tipo de discusión. No solo es innecesario limitar el estudio de la argumentación a los puntos de vista descriptivos, sino que también es absolutamente indeseable, ya que en ciertos terrenos del discurso tal limitación daría rienda suelta a quienes no están para nada interesados en justificar sus puntos de vista ante otros. En política, por ejemplo, esto les ofrecería una coartada para abstenerse de tener que argumentar en favor de sus acciones y les daría la oportunidad de volver sus puntos de visa inmunes a la crítica, al declararlos más allá de toda discusión. Porque, en cada decisión tomada en conjunto, no solo en política sino también en otras áreas de la esfera pública e incluso en la esfera privada, las discusiones argumentativas juegan $-\mathrm{o}$ deberían jugar- un papel crucial. Si tomamos este proceso de decisión seriamente, todos los casos en los que la argumentación se utilice para defender un punto de vista requerirán de un análisis cuidadoso y de una evaluación crítica 
del discurso argumentativo, y el estudio de la argumentación deberá suministrar las herramientas conceptuales necesarias.

Al tratarse de la noción pivote de argumentación, es posible observar algunas diferencias relevantes entre la palabra "argumentation" en inglés y los significados de sus contrapartes en otras lenguas europeas. Dado que estas diferencias pueden tener consecuencias significativas en la manera como se conceptualiza la argumentación, es importante tomar nota de ellas. Para empezar, a diferencia del término en inglés, la palabra holandesa "argumentatie" es, como su equivalente en muchas otras lenguas, ${ }^{3}$ una palabra muy común, que todo el mundo conoce y usa de la misma forma inequívoca. Más importante aún, los hablantes ordinarios utilizan esta palabra básicamente de la misma manera que los teóricos de la argumentación.

La primera propiedad que es necesario resaltar a propósito de la palabra holandesa "argumentatie" y de sus equivalentes en otras lenguas, es que se relaciona de inmediato con la idea de tratar de resolver una diferencia de opinión de forma constructiva, al convencer a la otra parte de la aceptabilidad del punto de vista propio - característica que tiene en común con el término teórico argumentación (van Eemeren y Grootendorst, A Systematic Theory of Argumentation, 11-18). A diferencia de la palabra "argumentation" en inglés, la palabra holandesa para argumentación no tiene nada que ver con la idea de pelear o con cualquier otra actividad verbal cargada negativamente, como reñir, discutir, disputar, contender y enemistarse. Esta ausencia de toda connotación negativa permite, al llevar a cabo una teorización, poder adoptarla como un término técnico, sin tener que presentar primero ciertas condiciones artificiales.

Una segunda propiedad que distingue la palabra holandesa para argumentación de la correspondiente inglesa, es el hecho de que la primera solo hace referencia a la constelación de razones expuestas por el que argumenta en defensa de su punto de vista y no al punto de vista mismo. Esta distinción es similar a la distinción lógica entre las premisas y la conclusión.

Una tercera propiedad distintiva es que la ambigüedad entre el proceso y el producto que caracteriza al término teórico argumentación, es

\footnotetext{
${ }^{3}$ Como es el caso del español [nota de la traductora].
} 
ya inherente al sentido de la palabra holandesa "argumentatie", mientras que este no es claramente el caso para el empleo ordinario de la palabra inglesa "argumentation".

Conceptualmente, el significado léxico de las contrapartes de la palabra "argumentation" constituye una mejor base para una definición teórica del término teórico de argumentación, que el significado de la palabra inglesa "argumentation". ¿Qué otras características conspicuas de la argumentación deben entrar en esta definición, dado que estas deben tener consecuencias metodológicas en la manera como se conduce la investigación sobre la argumentación? Permítaseme resumir las cuatro características que me parecen vitales.

Primero, la argumentación es un acto comunicativo complejo, que se lleva a cabo en la comunicación ordinaria por medio de un comportamiento funcional verbal (y, a veces, no verbal). En la teorización esta característica lleva a adoptar el principio metodológico de la "funcionalización".

Segundo, la argumentación es un acto interaccional complejo dirigido a otras personas, lo que hace que la argumentación forme parte de un diálogo explícito o implícito. El principio metodológico correspondiente es la "socialización".

Tercero, la argumentación implica emitir, de una u otra manera, proposiciones que involucran compromisos de los que el hablante puede ser considerado responsable. El principio metodológico respectivo es la "externalización".

Cuarto, debido a su naturaleza constructiva, la argumentación involucra un llamado a la sensatez, ${ }^{4}$ el cual deriva su fuerza de la idea de que hay estándares críticos comunes. Esto lleva a adoptar el principio metodológico de la "dialectificación".

Los principios metodológicos que acabo de mencionar son metateóricos, en el sentido de que su adopción precede la teorización misma. Son parte integrante del enfoque teorético de la argumentación del que parto, pero esto no significa que todos los teóricos de la argumentación los compartan.

Desde nuestro enfoque, la funcionalización se lleva a cabo partiendo del hecho de que el discurso argumentativo tiene lugar a través de $-\mathrm{y}$ en

${ }^{4}$ En inglés, el autor lo denomina “an appeal to reasonableness" [nota de la traductora]. 
respuesta a - la realización de actos de habla. Identificar el acto complejo de habla argumentativo y los otros actos de habla involucrados en la resolución de una diferencia de opinión, hace posible especificar las "condiciones de identidad" y las "condiciones de corrección" relevantes en estos actos de habla (van Eemeren y Grootendorst, Speech Acts in Argumentative Discussions, 39-46; Argumentation, Communication, and Fallacies, 30-33). De esta manera, por ejemplo, es posible especificar lo que está "en juego" al proponer un determinado "punto de vista", lo que aclara cuál es el "espacio de desacuerdo" y cómo se organiza el discurso argumentativo alrededor de este contexto de desacuerdo (Jackson, “"Virtual Standpoints' and the Pragmatics of Conversational Argument", 261).

La socialización se lleva a cabo al identificar quiénes en particular asumen los roles de protagonista y antagonista de la discusión, en el contexto de colaboración del discurso argumentativo. Al extender la perspectiva del acto de habla al nivel de la interacción, se puede mostrar de qué manera se desarrollan las posiciones y la argumentación a favor de estas posiciones.

La externalización se lleva a cabo al identificar los compromisos específicos que crean los actos de habla realizados en un contexto de interacción argumentativa. En lugar de considerarlos como estados mentales internos, en la perspectiva de los actos de habla, nociones como el "desacuerdo" y la "aceptación" pueden definirse en términos de actividades discursivas con compromisos resultantes bien definidos. La "aceptación", por ejemplo, puede externalizarse como el hecho de dar una respuesta preferente a un acto discutible que compromete al destinatario a dejar de atacar el acto en cuestión.

Por último, la dialectificación se lleva a cabo al reglamentar el intercambio de actos de habla dirigidos a resolver una diferencia de opinión, en un modelo ideal de la discusión crítica.

Al tomar en cuenta estos puntos de partida meta-teóricos, la argumentación puede definirse de la siguiente manera:

la argumentación es un acto (de habla) comunicativo e interaccional complejo, dirigido a resolver una diferencia de opinión para un juez razonable, proponiendo una constelación de razones de las que el argumentador 
puede considerarse responsable, para justificar la aceptabilidad del (o de los) punto(s) de vista en cuestión.

\section{La teoría pragma-dialéctica de la argumentación}

Los especialistas de la argumentación con frecuencia se sienten atraídos por este campo de estudio, debido a un interés en ciertas prácticas particulares del discurso argumentativo y en mejorar su calidad cuando así es necesario. Para poder satisfacer este interés tienen que combinar una orientación empírica con una orientación crítica hacia el discurso argumentativo. Esta desafiante combinación solo puede lograrse si no solo examinan el discurso argumentativo como un espécimen de la comunicación verbal y de la interacción reales, sino que también midan su calidad frente a estándares normativos de lo razonable. Si se considera que la "pragmática" es el estudio del uso comunicativo e interactivo del lenguaje, como se acostumbra entre los analistas del discurso, entonces la necesidad de unir el ángulo empírico y el descriptivo de la investigación, así como el ángulo crítico y el normativo puede satisfacerse, al tomar el estudio de la argumentación como una rama de la "pragmática normativa" (van Eemeren, "Dialectical Analysis as a Normative Reconstruction of Argumentative Discourse").

En pragmática normativa, los especialistas de la argumentación consideran que su tarea es explicar cómo se puede sistemáticamente establecer un enlace entre la dimensión normativa y la dimensión descriptiva de la argumentación, de manera que puedan integrarse la percepción crítica y la empírica. Los complejos problemas que están en juego en este esfuerzo solo pueden resolverse con la ayuda de un amplio programa de investigación que comprenda varios componentes interrelacionados (van Eemeren y Grootendorst, A Systematic Theory of Argumentation, 9-41). Por un lado, hay un componente filosófico en el programa donde debe desarrollarse una filosofía de lo razonable y un componente teórico en el que, partiendo de ese ideal de lo razonable, se diseñe un modelo de la argumentación aceptable. Por el otro, hay un componente empírico en donde debe investigarse, tanto cualitativa como cuantitativamente, la realidad argumentativa tal como se encuentra en el discurso 
argumentativo. Posteriormente, en el componente analítico es necesario unir sistemáticamente las dimensiones normativa y descriptiva. Por último, en el componente práctico se deben identificar los problemas que tienen lugar en las prácticas argumentativas particulares y desarrollar métodos para resolverlos.

Ahora bien, sucede que las concepciones de la argumentación razonable que han desarrollado los especialistas, a partir del componente filosófico de su programa de investigación, son desde un principio divergentes, de modo que en el componente teórico emergen diversas perspectivas de lo que se considera un argumento aceptable. Al desarrollar nuestro enfoque de la argumentación, Rob Grootendorst y yo estuvimos profundamente influenciados por la "dialéctica formal" de Barth y Krabbe (From Axiom to Dialogue), y partimos de una concepción de lo razonable que reemplaza el "justificacionismo" por un procedimiento crítico de evaluación (van Eemeren y Grootendorst, Speech Acts in Argumentative Discussions, 15-18). Esta concepción crítica y dialéctica de lo razonable se asocia con la filosofía "crítica racionalista" (popperiana) de lo razonable, la cual sostiene que, en última instancia, no podemos estar seguros de nada, y toma como principio guía la idea de evaluar críticamente toda afirmación sobre la aceptabilidad (van Eemeren y Grootendorst, "Rationale for a Pragma-Dialectical Perspective"). Como ha enfatizado Albert (Traktat über kritische Vernunft), la concepción crítica racionalista de lo razonable lo engloba todo: se refiere a cualquier tema que pueda ser objeto de una discusión regulada y cubre - como preferimos considerarlo- la discusión de puntos de vista tanto descriptivos como evaluativos y prescriptivos.

$\mathrm{Al}$ implementar la perspectiva crítica racionalista en el componente teórico del programa de investigación, buscamos desarrollar un modelo de discusión crítica que apoyara la idea de resolver las diferencias de opinión de fondo, por medio de intercambios críticos regulados dialécticamente, en los que se evalúa la aceptabilidad de los puntos de vista en juego (van Eemeren y Grootendorst, "Rationale for a Pragma-Dialectical Perspective", 279-280). El resultado de la discusión entre el protagonista y el antagonista depende de las preguntas críticas hechas por el antagonista y de la adecuación de las respuestas del protagonista a estas preguntas críticas. La interacción sistemática que tiene lugar, entre los 
actos de habla realizados por el protagonista para defender su punto de vista y aquellos del antagonista para responder críticamente, es característica del procedimiento de resolución "pragma-dialéctico" que hemos diseñado, el cual combina una perspectiva dialéctica de lo razonable argumentativo con una perspectiva pragmática de los movimientos verbales efectuados en el discurso argumentativo.

El modelo de una discusión crítica que proponemos ofrece una visión de conjunto de los movimientos argumentativos que son pertinentes para desarrollar cada una de las etapas de la discusión, que promueven el proceso para resolver una diferencia de opinión de fondo en cada etapa en particular. Analíticamente, en una discusión crítica se distinguen cuatro etapas que deben cumplirse de manera constructiva para poder resolver la diferencia de opinión de fondo. Primero, se encuentra la "etapa de confrontación" en la que se externaliza la diferencia de opinión desde el espacio de desacuerdo potencial. Después encontramos la "etapa de apertura", en la que el protagonista y el antagonista del punto de vista en cuestión determinan su zona de acuerdo, en cuanto a un procedimiento común y a puntos de partida materiales (o "concesiones"). En la "etapa argumentativa" ambas partes tratan de establecer si, una vez dado el punto de partida reconocido por las partes, el punto de vista del protagonista es sostenible teniendo en cuenta las respuestas críticas del antagonista. Por último, en la "etapa conclusiva", se establece el resultado de la discusión crítica.

El modelo pragma-dialéctico de una discusión crítica es un sistema motivado teóricamente enfocado en el discurso que se orienta hacia una resolución. En una discusión crítica, las partes intentan llegar a un acuerdo respecto a la aceptabilidad de los puntos de vista en juego, al averiguar si estos puntos de vista pueden o no ser defendibles frente a dudas o críticas. Para lograr este propósito, el procedimiento dialéctico para conducir una discusión crítica no puede ocuparse solo de relaciones de inferencia entre premisas (o "concesiones") y conclusiones (o "puntos de vista"), sino que debe incluir todos los actos de habla que tengan una función al examinar la aceptabilidad de los puntos de vista. En pragma-dialéctica, se le da forma al concepto de discusión crítica en un modelo que especifica todos los tipos de actos de habla instrumentales, en cualquiera de las etapas por las que debe pasar el proceso 
de resolución. Dado que en el discurso argumentativo real los actos de habla frecuentemente se realizan implícita o indirectamente, en la práctica, una gran variedad de actos de habla puede cumplir una función constructiva en el proceso de resolución (van Eemeren y Grootendorst, Speech Acts in Argumentative Discussions; A Systematic Theory of Argumentation).

\section{El tratamiento pragma-dialéctico de las falacias}

\subsection{Críticas al "tratamiento lógico estándar" de las falacias}

La prueba de fuego para cualquier teoría normativa de la argumentación es mostrar hasta qué punto nos permite dar cuenta de las falacias. Como todos sabemos, en el estudio de la argumentación, las falacias han sido un objeto de estudio importante desde la Antigüedad. Aristóteles las examinó ampliamente en sus estudios tanto dialécticos como retóricos. En los Tópicos, su tratado sobre la dialéctica, Aristóteles ubica las falacias en el contexto de un debate entre un atacante y el defensor de una tesis, en el que el atacante ataca y el defensor defiende la tesis. El atacante puede ganar el debate, en primer lugar, refutando la tesis del defensor. Aristóteles discute los movimientos correctos que puede efectuar el atacante para refutar la tesis del defensor, así como los movimientos incorrectos que considera falaces. En términos generales, en la perspectiva dialéctica de Aristóteles, las falacias son movimientos falsos que emplea el atacante en su esfuerzo por refutar la tesis del defensor. En las Refutaciones Sofísticas, Aristóteles se ocupa de las modalidades erróneas de refutar una tesis, que atribuía a los expertos populares del debate conocidos como los Sofistas — de ahí el epíteto "sofisma". En su Retórica, Aristóteles discute, desde una perspectiva retórica, algunas refutaciones falaces que solo son refutaciones aparentes.

Desde entonces, las falacias han sido un tema popular de estudio que, con el tiempo, ha llevado al descubrimiento de un número de "nuevas" falacias. Estas falacias recién descubiertas apenas fueron agregadas a la lista aristotélica de falacias dialécticas a pesar de que, gracias en gran medida a la considerable influencia del obispo Whatley, una perspectiva 
lógica mucho más amplia había gradualmente reemplazado la perspectiva dialéctica. Los nombres en latín que recibieron muchas de estas falacias pueden sugerir que todas vienen de la tradición clásica, pero no es así: por ejemplo, sin llamarlo falaz, John Locke llamó la atención sobre el argumentum ad hominem.

En 1970, Charles Hamblin revolucionó el estudio de las falacias con su monografía Fallacies, en la que cuenta su sorpresa al observar las similitudes en el tratamiento de las falacias en los principales libros de texto de la época. Observó que todos estos presentaban más o menos la misma lista de falacias, las explicaban más o menos de la misma forma, utilizando con frecuencia exactamente los mismos ejemplos. Hamblin sospechó que cada autor simplemente copiaba al otro sin ninguna reflexión suplementaria. Asimismo señaló que el "tratamiento lógico estándar" que detectó en los libros de texto, partía de una "definición lógica estándar" de las falacias en tanto que argumentos que parecen válidos pero que de hecho no lo son. Curiosamente, sin embargo, el tratamiento de las falacias era en muchos aspectos inconsistente con esta definición.

En primer lugar, muchas de las falacias consideradas en los libros de texto de lógica, como el argumentum ad hominem, no eran en realidad argumentos. O bien, se trataba de argumentos como el "razonamiento circular", que ciertamente no era inválido y también había otros, como el argumentum ad verecundiam, en el que la razón de su falsedad era completamente diferente a la invalidación.

La revelación de estas incongruencias condujo a mucha confusión, aun cuando los teóricos más abiertos reconocieron que Hamblin tenía razón. Con el tiempo, llegaron a compartir gran parte de sus objeciones al "tratamiento lógico estándar" de las falacias. En la actualidad no quedan muchos teóricos de la argumentación que consideren la "validez lógica" como el único criterio aplicable a las falacias. Los especialistas tienden también a coincidir en que el empleo de una palabra como "parecer" en la definición del término falacia, como es el caso en la "definición lógica estándar", trae consigo una indeseable cantidad de subjetividad psicologizante. En realidad, un cierto argumento puede parecerle bueno a alguien pero ¿por qué habría de parecerme bueno a mí, si yo sé que no es válido o que es deficiente de alguna otra forma? 
Por más pertinentes que fueron las devastadoras críticas de Hamblin, no fueron siempre tan productivas en la práctica, como puede verse en las dos siguientes reacciones extremas. Para empezar, hubo autores de importantes libros de lógica, como Copi (Introduction to Logic), que reimprimieron sus estudios sobre las falacias sin intentar seriamente responder a las objeciones de Hamblin. Quizás pensaron que sus libros de texto funcionaban bien tal como estaban - por lo demás, ¿qué sabían sus estudiantes de Hamblin? La reacción extrema opuesta a las críticas de Hamblin fue abandonar por completo el tratamiento de las falacias en los libros de texto. Desde una perspectiva ética esto podría ser mejor, pero es claro que no contribuye a tratar el problema de las falacias. Una tercera opción que hubiera sido posible imaginar como una forma fácil de salir del problema, sería mantener la "definición lógica estándar" dejando fuera todas aquellas falacias que no respondieran a esta definición pero, afortunadamente, no hay muchos teóricos de la argumentación dispuestos a tirar al niño con el agua de bañarlo, y a hacer a un lado sus esfuerzos de encontrar un tratamiento apropiado para las falacias, con el único objeto de mantener cierta pureza teórica.

\subsection{Una alternativa constructiva al "tratamiento lógico estándar"}

Desde mi punto de vista, la teorización sobre las falacias debe partir de una perspectiva general y coherente sobre el discurso argumentativo que provea de fundamentos comunes para todos los estudios de las falacias. Dado que una teoría de lo incorrecto no puede construirse independientemente de una teoría de lo que es normativamente correcto, una teoría sobre las falacias debe ser parte integral de una teoría normativa de la argumentación que suministre estándares bien definidos de lo que es un discurso argumentativo razonable. La explicación teórica de las falacias debe estar sistemáticamente relacionada con estos estándares, de tal manera que sea claro en todos los casos por qué las falacias son falaces.

En Europa, a principios de los ochenta, se desarrollaron dos aproximaciones dialécticas a la argumentación que constituyen una secuela constructiva de las críticas de Hamblin, en las que este autor relaciona 
sistemáticamente las falacias a los estándares de una argumentación razonable. Ambos enfoques estaban dirigidos a desarrollar una teoría de la argumentación que se inicia en una perspectiva "crítica racionalista" sobre el discurso argumentativo, en el que la falibilidad de todo el pensamiento humano es el punto de partida: la dialéctica formal desarrollada por Else Barth y Erik Krabbe (From Axiom to Dialogue) y la teoría pragma-dialéctica de la argumentación que desarrollé con Rob Grootendorst (van Eemeren y Grootendorst, Speech Acts in Argumentative Discussions; Argumentation, Communication, and Fallacies; A Systematic Theory of Argumentation), y que posteriormente he profundizado con Peter Houtlosser (van Eemeren y Houtlosser, "Strategic Maneuvering"; "Fallacies as Derailments"; "More about Fallacies"). Dado que me centro en la identificación de las falacias en el discurso ordinario, utilizaré la pragma-dialéctica como marco teórico, en lugar de la dialéctica formal. La pragma-dialéctica se vincula a la dialéctica formal, pero considera el discurso argumentativo y las falacias que surgen en el discurso argumentativo — pragmáticamente - en una perspectiva comunicativa e interaccional.

La situación argumentativa más simple es aquella de un hablante o escritor que presenta un punto de vista y que actúa como "protagonista" de dicho punto de vista, y la de un oyente o lector que expresa duda en relación con el punto de vista, actuando como "antagonista". En la discusión que se desarrolla, ambas partes tratan de averiguar si el punto de vista del protagonista puede resistir las críticas del antagonista. En este intercambio se lleva a cabo una interacción entre los actos de habla realizados por el protagonista y los que realiza el antagonista, interacción típica de lo que llamamos una "discusión crítica". Esta interacción puede, desde luego, llevar hacia la resolución de la diferencia de opinión solo si procede de forma adecuada, lo que requiere que se regule a través de las reglas para la discusión crítica que especifican en qué casos la realización de ciertos actos de habla contribuye a la resolución de la diferencia de opinión de fondo. La tarea de los teóricos de la argumentación dialéctica es la de formular estas reglas de tal manera que en conjunto constituyan un procedimiento de discusión que sea válido, tanto para resolver problemas, como desde un punto de vista convencional (Barth y Krabbe, From Axiom to Dialogue, 21-22). Las reglas de 
procedimiento que propone la pragma-dialéctica se consideran útiles ante los problemas, porque cada una de ellas contribuye, de una cierta manera, a solucionar los que son inherentes al proceso de resolver una diferencia de opinión. Su validez convencional se confirma con la investigación sistemática experimental sobre su aceptabilidad intersubjetiva (van Eemeren, Garssen y Meuffels, The Intersubjective Validity of the Rules for Critical Discussion).

Un procedimiento que regule la resolución de una diferencia de opinión no puede confinarse exclusivamente a las relaciones lógicas por medio de las cuales se infieren conclusiones a partir de premisas. Como es de esperarse, debe consistir en un sistema de reglas que incluya todos los actos de habla que es necesario realizar para resolver una diferencia de opinión. Esto quiere decir que el procedimiento debe relacionarse con las cuatro etapas que se distinguen en una discusión crítica: las reglas para llevar a cabo una discusión crítica deben indicar todas las normas pertinentes para resolver una diferencia de opinión de fondo. En principio, cada una de las reglas de la discusión pragma-dialéctica constituye una norma distinta para la discusión crítica. Cualquier movimiento que sea una infracción de una de estas reglas, sin importar la parte que lo realice o la etapa de la discusión en que suceda, es una amenaza potencial para la resolución de una diferencia de opinión y debe por lo tanto - y en este sentido particular - ser considerado como falacioso. De esta manera, el uso del término falacia está sistemáticamente relacionado con las reglas de la discusión crítica. En la perspectiva pragma-dialéctica, una falacia es entonces un obstáculo o impedimento para resolver una diferencia de opinión de fondo. La naturaleza específica de una falacia particular depende de la manera como interfiere con el proceso de resolución.

El enfoque pragma-dialéctico distingue una variedad funcional de normas para juzgar lo que es falaz. En lugar de considerar las falacias como parte de una lista no estructurada de categorías nominales que heredamos del pasado, como lo hace el "tratamiento lógico estándar", o de considerar todas las falacias como violaciones de la norma de validez (como en los acercamientos centrados en la lógica), hay diferentes (combinaciones de) normas que pueden ser pertinentes. Una comparación muestra que las falacias que tradicionalmente solo fueron no- 
minalmente agrupadas, ahora evidencian tener aspectos en común o distinguirse claramente entre sí, mientras que falacias que están genuinamente relacionadas entre sí y antes se encontraban separadas, vuelven a ser reagrupadas. Hay, por ejemplo, dos tipos diferentes de argumentum ad populum, la falacia de juzgar algo aceptable porque muchas personas así lo consideran; una de las variantes es una violación de la "regla de relevancia", según la cual una parte puede defender su punto de vista solo presentando una argumentación relacionada con dicho punto de vista; la otra variante es una violación de la "regla del esquema argumentativo", de acuerdo con la cual un punto de vista no puede considerarse como habiendo sido defendido de forma concluyente, si la defensa no se lleva a cabo por medio de un esquema argumentativo apropiado, es decir, correctamente empleado. Entre las falacias que fueron separadas y que se han reagrupado, hay una variante del argumento ad verecundiam (utilizar un esquema argumentativo sintomático inapropiado, presentando el punto de vista como correcto porque una autoridad afirma que es correcto) y una variante del ad populum (emplear un esquema argumentativo sintomático inapropiado, presentando el punto de vista como correcto porque todo el mundo piensa que es correcto). Cuando son analizadas como violaciones de la misma "regla del esquema argumentativo", vemos claramente que desde la perspectiva de la resolución de una diferencia de opinión, estas variantes son básicamente del mismo tipo.

De manera adicional, el acercamiento pragma-dialéctico también permite analizar "nuevos" obstáculos, que aún no han sido reconocidos ni identificados, para resolver una diferencia de opinión de fondo. Ejemplos de lo anterior son: el declarar un punto de vista sacrosanto, que corresponde con una violación de la "regla de libertad", la cual dice que las partes no pueden impedirse mutuamente presentar puntos de vista o formular dudas respecto a ellos; el evadir la carga de la prueba y cambiar la carga de la prueba, ambas son violaciones a la "regla de la carga de la prueba", según la cual, la parte que presenta un punto de vista está obligada a defenderlo si se le solicita; el negar una premisa no expresada, que corresponde con una violación a la "regla de la premisa no expresada": una parte no puede falsamente presentar como premisa algo que no se ha explicitado, o negar una premisa que 
se ha dejado implícita; y el hacer un absoluto del éxito de la defensa que equivale a una violación de la "regla del cierre", que dice que una defensa fallida solo puede resultar en que el protagonista se retracte de su punto de vista y una defensa exitosa en que el antagonista se retracte de su duda (van Eemeren y Grootendorst, Argumentation, Communication, and Fallacies).

\section{Las falacias como descarrilamientos de las maniobras estratégicas}

A pesar de que puedo afirmar con seguridad que las críticas de Hamblin ya no se aplican a la teoría pragma-dialéctica de las falacias que acabo de esbozar, desde mi punto vista, esta teoría todavía no es enteramente satisfactoria. La razón principal es que ignora el problema intrigante de la persuasión que pueden producir estas falacias, razón por la cual, de hecho, merecen nuestra atención. Es verdad que el "meta-análisis" de estudios de persuasión expermimentales de Daniel O’Keefe ("Pragma-Dialectics and Persuasion Effect Research") parece sugerir que, en general, la argumentación razonable es más persuasiva que la argumentación falaz, y los resultados de nuestra propia investigación empírica apuntan en la misma dirección (van Eemeren, Garssen y Meuffels "Reasonableness in Confrontation"). Sin embargo, la discreta fuerza persuasiva de las falacias es un asunto tan espinoso que no podemos contentarnos con estas indicaciones, sino que debemos darle a este asunto nuestra completa atención.

En la "definición lógica estándar" de las falacias como "argumentos que parecen válidos pero que no lo son", la capacidad persuasiva de las falacias se insinuaba al emplear la palabra "parecen", pero ya que Hamblin (Fallacies, 254) emitió el veredicto de que incluir esta calificación acarrea un elemento de subjetividad indeseado, el carácter traicionero de las falacias - la palabra fallax en latín significa engañoso o falsoha sido ignorado y se abandonó la búsqueda de su explicación. Esto quiere decir que a los teóricos de las falacias ya no les preocupa la cuestión de cómo "funcionan" las falacias, es decir, cómo pueden ser exitosas y por qué pasan con frecuencia desapercibidas. Creo que la teoría 
pragma-dialéctica de la argumentación puede remediar este abandono pero, debido a la naturaleza del problema, solo puede hacerlo si primero se enriquece con la percepción de la retórica.

En esta coyuntura, es importante hacer hincapié en que combinar la percepción retórica y la dialéctica es mucho más problemático de lo que uno podría pensar. A pesar de su conexión inicial en la Antigüedad, cuando Aristóteles describió la retórica como la imagen del espejo (antistrophos) o la contraparte de la dialéctica, desde la revolución científica del siglo XVII — empezando, de hecho, ya con Ramus - se ha dado una tajante división ideológica entre la dialéctica y la retórica. Esta división ha resultado en la existencia de dos paradigmas separados y mutuamente aislados que se conforman en perspectivas diferentes sobre la argumentación, generalmente consideradas como incompatibles. La retórica se convirtió en el campo de estudios para los especialistas en comunicación, literatura y lengua en las humanidades y las ciencias sociales, mientras que la dialéctica pasó a ser el terreno de los especialistas en lógica y de los científicos — pero casi se perdió de vista después de la formalización de la lógica a finales del siglo XIX y principios del xx. A pesar de que el enfoque dialéctico de la argumentación fue retomado por Hamblin y sus seguidores en la segunda mitad del siglo $\mathrm{xx}$, durante mucho tiempo hubo - $\mathrm{y}$, en gran medida, sigue habiendoun enorme abismo conceptual y comunicativo entre los teóricos de la argumentación que optan por la perspectiva dialéctica y los teóricos de la argumentación con una perspectiva retórica. En las dos últimas décadas, sin embargo, se han llevado a cabo grandes esfuerzos por superar la tajante e infértil división entre la dialéctica y la retórica (van Eemeren y Houtlosser, "Strategic Maneuvering in Argumentative Discourse").

La inclusión de la percepción retórica en la teoría pragma-dialéctica que Peter Houtlosser y yo hemos impulsado, es un ejemplo claro de un esfuerzo por cerrar la brecha entre la dialéctica y la retórica (van Eemeren y Houtlosser, "Strategic Maneuvering", "Fallacies as Derailments", "More about Fallacies", "Theoretical Construction"). Comenzamos observando que en el discurso argumentativo, ya sea que se trate del discurso oral o del escrito, el único objetivo de los argumentadores no es el de llevar la discusión de una forma que pudiera considerarse razonable, sino que también y desde cierta perspectiva, incluso en mayor medida, 
está el lograr el resultado que desde el punto de vista de cada argumentador sea el mejor. Los intentos retóricos de cada una de las partes por lograr que las cosas vayan a su manera, se incorporan a sus esfuerzos dialécticos por resolver la diferencia de opinión, de acuerdo con los estándares apropiados de una discusión crítica. Esto significa, en la práctica, que en cada etapa del proceso de resolución se puede suponer que las partes buscan, al mismo tiempo, lograr el resultado retórico óptimo en ese punto de la discusión y mantener el objetivo dialéctico que corresponde a esa etapa de la discusión. En sus intentos por reconciliar la búsqueda simultánea de estos dos objetivos, que a veces pueden estar en conflicto, los argumentadores utilizan lo que hemos llamado maniobras estratégicas. Estas maniobras estratégicas buscan disminuir la tensión potencial entre la búsqueda simultánea del objetivo "dialéctico" de lo razonable y del objetivo "retórico" de lo efectivo.

Las maniobras estratégicas se manifiestan en los movimientos que se hacen en el discurso argumentativo de acuerdo con tres aspectos, que pueden distinguirse solo de forma analítica: la "elección del tópico", la "adaptación al auditorio" y el "diseño de presentación". La elección del tópico se refiere a la selección específica que se hace en cada movimiento a partir del potencial de tópicos - el conjunto de opciones dialécticas - disponible en la etapa que corresponde de la discusión; la adaptación al auditorio involucra enmarcar los movimientos propios en una perspectiva con la que el auditorio esté de acuerdo; el diseño de presentación se refiere a la selección que hace el que habla o escribe con respecto al repertorio existente de dispositivos de presentación. Al llevar a cabo sus maniobras estratégicas, dirigidas a conducir el discurso argumentativo a su favor sin violar ningún estándar crítico en el proceso, puede considerarse que ambas partes están interesadas en hacer la selección tópica más conveniente, para atraer con la mayor fuerza a su auditorio, adoptando la presentación más efectiva.

Es posible lograr una mejor comprensión de las maniobras estratégicas en el discurso argumentativo, si examinamos cómo se explotan en la práctica argumentativa las oportunidades retóricas disponibles en una situación dialéctica. Cada una de las cuatro etapas del proceso para resolver una diferencia de opinión se caracteriza por tener un objetivo dialéctico específico. Dado que, como es de esperarse, las partes desean 
alcanzar estos objetivos para el mejor aprovechamiento de la postura que han adoptado, cada objetivo dialéctico tiene su análogo retórico. En cada etapa de la discusión, los objetivos retóricos de los participantes dependerán de $-\mathrm{y}$, por lo tanto, irán paralelos a- sus objetivos dialécticos, porque en cada etapa están tratando de lograr los resultados dialécticos que sirvan mejor a sus propósitos retóricos. Como consecuencia, las especificaciones de los objetivos retóricos que pueden atribuírseles a los participantes en el discurso, deben llevarse a cabo de acuerdo con la etapa dialéctica. Esta es la razón metodológica por la que el estudio de las maniobras estratégicas que proponemos se reduce a una integración sistemática del conocimiento retórico en un marco dialéctico - en nuestro caso, pragma-dialéctico- del análisis.

El tipo de ventajas que puede lograrse a través de las maniobras estratégicas depende de la etapa particular en la que uno se encuentre. En la etapa de confrontación, por ejemplo, el objetivo dialéctico es el de lograr claridad en relación con las cuestiones que están en juego y las posiciones que asumen las partes. Las maniobras estratégicas de cada parte estarán por lo tanto orientadas a dirigir la confrontación retóricamente, hacia una definición de la diferencia que resalte precisamente las cuestiones que cada parte quiere discutir. En la etapa de apertura, el objetivo dialéctico es el de establecer un comienzo inequívoco que consista en puntos de partida, tanto de procedimiento como materiales, intersubjetivamente aceptados. Como consecuencia, las maniobras estratégicas de las partes estarán dirigidas a establecer retóricamente puntos de partida de procedimiento, que aseguren una distribución oportuna de la carga de la prueba, y que combinen el tener reglas de discusión deseables con puntos de partida materiales que impliquen concesiones útiles por parte del otro. En la etapa de la argumentación, cuando se cuestionan y defienden los puntos de vista en juego, el objetivo dialéctico es el de probar, empezando por el punto de partida establecido en la etapa de apertura, la sustentabilidad de los puntos de vista que conformaron la diferencia de opinión en la etapa de confrontación. Dependiendo de las posiciones tomadas, las partes realizarán maniobras estratégicas para ingeniar retóricamente el caso más convincente —o bien el ataque más efectivo, según sea el caso. En la etapa de conclusión, el objetivo dialéctico de determinar si, y a favor de quién, se ha resuelto la diferencia de 
opinión, lleva a maniobras estratégicas dirigidas a reforzar la victoria a favor de la parte preocupada por lograr retóricamente, ya sea la conclusión de que el protagonista puede mantener su punto de vista, tomando en cuenta las críticas que se hicieron, o que el antagonista puede mantener su duda, en vista de la argumentación presentada.

A pesar de que, desde nuestro punto de vista, en las maniobras estratégicas la búsqueda de objetivos dialécticos es compatible con la realización de objetivos retóricos, esto desde luego no quiere automáticamente decir que al final los dos objetivos siempre se encontrarán en un balance perfecto. Si una de las partes permite que su compromiso de llevar a cabo un intercambio crítico de movimientos argumentativos se llegue a invalidar por el objetivo de persuadir al oponente, podemos decir que las maniobras estratégicas se "descarrilaron". Tales descarrilamientos ocurren cuando se viola una regla para la discusión crítica. En ese caso, tratando de lograrse, el objetivo retórico toma la delantera - a expensas de conseguir el objetivo dialéctico. Dado que los descarrilamientos de las maniobras estratégicas siempre involucran violar alguna regla para la discusión crítica, van a la par con los movimientos erróneos en el discurso argumentativo llamados falacias. Vistas desde esta perspectiva, las falacias son descarrilamientos de las maniobras estratégicas que involucran violaciones de las reglas de la discusión crítica.

La diferencia entre las manifestaciones legítimas de las maniobras estratégicas y las manifestaciones falaces es que, en estas últimas, no se cumplieron ciertas condiciones de sensatez que se aplican a esa forma de maniobra estratégica. Cada modalidad de maniobra estratégica tiene, digamos, un contínuum propio de actuación razonable y falaz, y los límites entre ambos no siempre resultan de inmediato claros. En la mayoría de las veces, los juicios falaces acaban siendo juicios contextuales que dependen de las circunstancias específicas de una actuación argumentativa localizada. El criterio para determinar si una cierta norma para la discusión crítica ha sido violada o no puede depender de las convenciones institucionales del "tipo de actividad argumentativa" en cuestión, esto es, de cómo es posible disciplinar el discurso argumentativo en un tipo particular de caso - haciendo referencia al precedente, por ejemplo, un llamado a la autoridad puede perfectamente ser legítimo en un caso judicial, mas no lo sería tan fácilmente en una discusión científica. 
Desde luego que esto no quiere decir, automáticamente, que no haya criterios claros para determinar si la maniobra estratégica perdió el camino, sino solo que la forma específica que toman estos criterios puede, hasta cierto punto, variar de un tipo de actividad argumentativa a otro.

Esta descripción de las falacias como descarrilamientos de las maniobras estratégicas explica por qué es posible que no sea inmediatamente aparente para todos los involucrados, como se esperaría, que una falacia haya sido cometida, pudiendo así pasar desapercibida. En principio, cada falacia tiene contrapartes razonables, que son manifestaciones de la misma modalidad de maniobra estratégica, de manera que es más difícil diferenciarlas de lo que sería distinguir dos tipos completamente diferentes de animales, como si todos los movimientos legítimos fueran gatos $\mathrm{y}$ todos los falaces fueran perros. Esto va enteramente en el mismo sentido de lo que Sally Jackson ("Fallacies and Heuristics") llama la asunción de lo razonable, según la cual, cuando una de las partes lleva a cabo maniobras estratégicas, normalmente defenderá su compromiso con las reglas de la discusión crítica, de manera que se le confiere la presunción de ser razonable en cada movimiento de la discusión - y esta suposición también opera cuando las maniobras estratégicas empleadas son falaces.

Las desviaciones de las reglas para la discusión crítica pueden ser difíciles de detectar porque ninguna de las partes estará dispuesta a mostrarse como irracional. Es de esperarse que para lograr un propósito que está potencialmente en conflicto con el objetivo de una determinada regla de la discusión, en lugar de recurrir a medios completamente diferentes, las partes se apegarán a los medios dialécticos usuales para lograr su objetivo, y "estirarán" el uso de estos medios hasta el punto que permitan también lograr este otro propósito. Haciéndonos eco de la "definición lógica estándar" de una falacia, podemos entonces decir que las maniobras estratégicas implicadas parecen cumplir con las reglas de la discusión crítica, pero de hecho no lo hacen.

\section{Argumentos de autoridad y el argumentum ad verecundiam}

Las falacias han sido, pues, caracterizadas como violaciones de las reglas para la discusión crítica, las cuales se manifiestan en descarrilamientos 
de las maniobras estratégicas que pueden fácilmente escapar a nuestra atención, porque dichos descarrilamientos pueden ser muy similares a instancias familiares de maniobras estratégicas razonables. De cualquier forma, es desde luego necesario hacer la distinción. Para marcar claramente la importancia de la distinción entre las maniobras estratégicas no falaces y las falaces, no utilizo indiscriminadamente los mismos términos para los movimientos falaces y para los no falaces, como lo hacen Walton y otros, sino que reservo los nombres tradicionales - con frecuencia latinizados - como el argumentum ad hominem, solo para los casos incorrectos y falaces.

Las maniobras estratégicas solo se descarrilan en falaces si van en contra de las normas para llevar a cabo un intercambio razonable, encarnado en las reglas para la discusión crítica. Esto quiere decir, en la práctica, que los movimientos argumentativos en cuestión no están de acuerdo con los criterios relevantes para cumplir con una determinada norma dialéctica. Como ya observamos, estos criterios están determinados por las condiciones de sensatez que tienen que cumplir los movimientos argumentativos, para mantenerse dentro de los límites de lo dialéctico razonable en el contexto argumentativo en el que se llevan a cabo, y que pueden, hasta cierto punto, variar de acuerdo con el tipo de actividad argumentativa en el que tienen lugar.

Como un caso puntual, evitando en lo posible el uso de un lenguaje técnico, discutiré brevemente la delimitación de los movimientos no falaces y de los falaces en un modo particular de maniobra estratégica que tiene lugar en un, digamos, amplio tipo de actividad argumentativa, el de una conversación informal. El modo de maniobra que he elegido consiste en defender un punto de vista presentando un "argumento de autoridad". El esquema argumentativo utilizado en un argumento de autoridad es un subtipo de la argumentación conocida como "argumentación sintomática", también llamada "argumentación del signo". En el esquema argumentativo, la argumentación de este tipo se basa en que la aceptabilidad de la premisa se presenta como signo de que la conclusión es aceptable, a través del establecimiento de una relación de concomitancia entre la propiedad mencionada en la premisa y la propiedad mencionada en la conclusión. Tal asociación sintomática fija aparece sugerida, por ejemplo, en una argumentación como "Paul debe ser un 
amante del queso porque es holandés", en donde se especifica que gustar del queso siempre lleva aparejado el ser holandés. En el caso del argumento de autoridad, la transición de aceptación está garantizada cuando se refiere, en la premisa, a una fuente externa que tiene el conocimiento o la experiencia requeridos para poder sacar la conclusión, de manera que tener el conocimiento se presenta como una señal de que las aseveraciones del experto son aceptables: "La capacidad para aprender una lengua es innata - de acuerdo con Chomsky".

Utilizar argumentos de autoridad es potencialmente una manera razonable de llevar a cabo una maniobra estratégica, como también lo es emplear otros argumentos del signo. En una gran cantidad de ocasiones es absolutamente justificado apoyar nuestras afirmaciones haciendo referencia a una autoridad que supuestamente conoce sobre el asunto tratado - en la práctica argumentativa esta es, de hecho, con mucha frecuencia la única cosa razonable por hacer. Si tenemos buenas razones para pensar que la fuente a la que nos referimos es efectivamente confiable en el caso de que se trata, y que debió ser tomada en cuenta cuando el argumentador hizo la observación referida, apelar a la autoridad puede no ser problemático e incluso puede ser conclusivo. Sin embargo, en la práctica argumentativa, las maniobras estratégicas por medio de argumentos de autoridad también pueden descarrilarse. Apelar a la autoridad puede no estar justificado en un determinado caso, debido a que alguna de las "preguntas críticas", necesarias para comprobar si los criterios de evaluación de los argumentos de autoridad en el tipo de actividad referida se han cumplido, puede no ser satisfactoriamente respondida, de manera que el argumento viola la "regla del esquema argumentativo" y debe considerarse un argumentum ad verecundiam.

En los diversos tipos de actividades argumentativas se pueden aplicar diferentes criterios para cumplir con la norma de sensatez incorporada a la variante argumento-de-autoridad de la "regla del esquema argumentativo". En el tipo de actividad informal de un intercambio conversacional que tomamos como nuestro contexto ilustrativo, los participantes son, en principio, quienes deciden cuáles serán las condiciones para llevar a cabo maniobras estratégicas razonables, por medio de argumentos de autoridad. Para nuestros propósitos de ejemplificación, distinguimos tres subtipos de intercambios conversacionales, cada uno caracterizado 
por su propia serie de convenciones "institucionalizadas". En el primer subtipo, (1a) las partes en el intercambio acordaron de antemano que apelar a una autoridad es legítimo, y (1b) el acuerdo permite apelar a un tipo de autoridad específico. Si en una práctica argumentativa del primer subtipo se cumplen las condiciones (1a) y (1b), no se comete un argumentum ad verecundiam al utilizar un argumento de autoridad, y utilizarlo puede ser considerado como una maniobra estratégica razonable. En el segundo subtipo, (2a) las partes implicadas en la discusión acordaron en la segunda instancia que una apelación a la autoridad es legítima y (2b) el acuerdo especifica con precisión a qué tipo de autoridad se puede apelar. Si en una práctica argumentativa del segundo subtipo se cumplen las condiciones (2a) y (2b) en la maniobra estratégica realizada, nuevamente, no se ha cometido un argumentum ad verecundiam y utilizar un argumento de autoridad puede considerarse una maniobra estratégica razonable. En el tercer subtipo (3), las partes implicadas en la discusión no llegaron a ningún acuerdo acerca de la legitimidad de apelar a la autoridad. Si en una práctica argumentativa del tercer subtipo se satisface la condición (3), no se ha violado ninguna regla de la discusión crítica, pero el uso de un argumento de autoridad puede perfectamente introducir su legitimidad como un nuevo tópico de discusión.

Los casos que acabo de discutir solo se refieren al acuerdo entre las partes sobre las condiciones para llevar a cabo maniobras estratégicas razonables relativas a argumentos de autoridad. En ciertos tipos de actividad argumentativa tal acuerdo auto-creado puede ser una condición suficiente; por ejemplo, cuando afirmo en un intercambio conversacional en ocasión de un juego de Scrabble, que la palabra que acabo de poner en el tablero es una palabra inglesa porque está en el Oxford Advanced Learners Dictionary, después de que tú y yo acordamos con anterioridad que ese iba a ser nuestro criterio para determinar si una palabra pertenecía o no al inglés. En otros tipos de actividades argumentativas, no obstante, los acuerdos relevantes estarán institucionalmente predeterminados e igualmente se tendrá que cumplir con otras condiciones. En una discusión científica, por ejemplo, la comunidad científica ("foro") determina qué fuentes cuentan como autoridades y qué otras condiciones aplican para poder recurrir a tal fuente. No es difícil imaginar, en relación con los argumentos de autoridad, que incluso otras condiciones 
sean válidas para realizar maniobras estratégicas razonables en otros tipos de actividades argumentativas, como en el debate parlamentario o en los editoriales de un periódico. Lo mismo sucede para otras modalidades de maniobras estratégicas, como al asignar la carga de la prueba, o señalar una inconsistencia en la posición de la otra parte, realizados en cualquiera de estos tipos de actividad argumentativa; de manera que en diferentes contextos argumentativos se aplicarán diversos criterios para cumplir con las reglas para la discusión crítica. Esto lleva la teoría de la argumentación de regreso al contexto del discurso argumentativo localizado, y este es exactamente el contexto en el que tanto Toulmin como Perelman y Olbrechts-Tyteca querían que se estudiara la argumentación.

\section{REFERENCIAS}

Albert, H., Traktat über kritische Vernunft, Tubingen, Mohr., 3ra ed., 1975. Aristóteles, Rhetoric, trad. W. D. Ross, Oxford, Clarendon Press, 1924.

Aristóteles, Sophistical Refutations, trad. y ed. W. D. Ross, Oxford, Clarendon Press, 1928.

Aristóteles, Topics, trad. y ed. W. D. Ross, Oxford, Clarendon Press, 1928.

Barth, E. M. y E.C.W. Krabbe, From Axiom to Dialogue: A Philosophical Study of Logics and Argumentation, Berlin-New York, Walter de Gruyter, 1982.

Biro, J. y H. Siegel, "Normativity, Argumentation and an Epipstemic Theory of Fallacies", en Argumentation Illuminated, F. H. van Eemeren, R. Grootendorst, J. A. Blair y Ch. A. Willard (eds.), Amsterdam, Sic Sat, 1992, 85-103.

Copi, I. M., Introduction to Logic, New York, Macmillan, 4ta ed., 1972.

Eemeren, F. H. van, "Dialectical Analysis as a Normative Reconstruction of Argumentative Discourse", Text: An Interdisciplinary Journal for the Study of Discourse, 6, 1986, 1-16.

Eemeren, F. H. van, Strategic Maneuvering in Argumentative Discourse. Extending the Pragma-Dialectical Theory of Argumentation. AmsterdamPhiladelphia, John Benjamins, 2010.

Eemeren, F. H. van, Maniobras estratégicas en el discurso argumentativo. Extendiendo la teoría Pragma-dialéctica de la argumentación, trad. C. Santibáñez, Madrid, Consejo Superior de Investigaciones Cientificas/Plaza y Valdés, 2011. 
Eemeren, F. H. van, B. Garssen y B. Meuffels, "Reasonableness in Confrontation: Empirical Evidence Concerning the Assessment of ad Hominem Fallacies", en Controversy and Confrontation: Relating Controversy Analysis with Argumentation Theory, F. H. van Eemeren y B. Garssen (eds.), Amsterdam-Philadelphia, John Benjamins, 2008, 181-195.

Eemeren, F. H. van, B. Garssen y B. Meuffels, The Intersubjective Validity of the Rules for Critical Discussion. Experimental Research, 2009 [en prensa].

Eemeren, F. H. van y R. Grootendorst, Speech Acts in Argumentative Discussions: A Theoretical Model for the Analysis of Discussions Directed towards Solving Conflicts of Opinion, Berlin-Dordrecht, De Gruyter/Foris, 1984 [versión española en preparación].

Eemeren, F. H. van y R. Grootendorst, "Rationale for a Pragma-Dialectical Perspective", Argumentation, 2, 1988, 271-291.

Eemeren, F. H. van y R. Grootendorst, Argumentation, Communication, and Fallacies: A Pragma-Dialectical Perspective, Hillsdale, New Jersey, Lawrence Erlbaum Associates, 1992.

Eemeren, F. H. van y R. Grootendorst, Argumentación, comunicación y falacias.Una perspectiva pragma-dialéctica [2002], C. López y A. M. Vicuña (trads.), Santiago, Ediciones Universidad Católica de Chile, 2007.

Eemeren, F. H. van y R. Grootendorst, A Systematic Theory of Argumentation: The Pragma-Dialectical Approach, Cambridge, Cambridge University Press, 2004.

Eemeren, F. H. van y R. Grootendorst, Una teoría sistemática de la argumentación. La perspectiva pragmadialéctica, C. López y A. M. Vicuña (trads.), Buenos Aires, Biblos y Universidad de Buenos Aires, 2011.

Eemeren, F. H. van, R. Grootendorst y A. F. Snoeck Henkemans Argumentación. Análisis, Evaluación, Presentación, trad. R. Marafioti, Buenos Aires, Biblos y Universidad de Buenos Aires, 2006.

Eemeren, F.H. van, R. Grootendorst, A.F. Snoeck Henkemans, J.A. Blair, R. H. Johnson, E. C. W. Krabbe, C. Plantin, D. N. Walton, C.A. Willard, J. Woods y D. ZAREFSKY, Fundamentals of Argumentation Theory, Mahwah, New Jersey, Erlbaum, 1996.

Eemeren, F. H. van y P. Houtlosser, "Strategic Maneuvering in Argumentative Discourse: A Delicate Balance", en Dialectic and Rhetoric: The Warp and Woof of Argumentation Analysis, F. H. van Eemeren, y P. Houtlosser, Dordrecht et al. (eds.), Kluwer Academic, 2002, 131-159.

Eemeren, F. H. van y P. Houtlosser, "Fallacies as Derailments of Strategic Maneuvering: The argumentum ad verecundiam, a Case in Point", en Proceedings of the Fifth Conference of the International Society for the Study of Argumentation, F. H. van Eemeren, J. A. Blair, C. A. Willard y A. F. Snoeck Henkemans (eds.), Amsterdam, Sic Sat, 2003, 289-292. 
Eemeren, F. H. van y P. Houtlosser, "More about Fallacies as Derailments of Strategic Maneuvering: The Case of tu quoque", en Argumentation and its Applications. InformalLogic@ @, H. V. Hansen, C. W. Tindale, J. A. Blair, R. H. Johnson y R. C. Pinto (eds.), CD ROM, 2004.

Eemeren, F. H. van y P. Houtlosser, "Theoretical Construction and Argumentative Reality: an Analytic Model of Critical Discussion and Conventionalised Types of Argumentative Activity", en The Uses of Argument. Proceedings of a Conference at McMaster University, 18-21 May 2005, D. Hitchcock y D. Farr (eds.), Hamilton, ON, McMaster University, 2005, 75-84.

FinOCCHIARO, M., "Mill's On Liberty and Argumentation Theory", en The Uses of Argument: Proceedings of a Conference at McMaster University May 18-21, 2005, D. Hitchcock (ed.), Hamilton, ON: Ontario Society for the Study of Argumentation, 2005, 89-98.

Hamblin, C. L., Fallacies, London, Methuen, Reimpresión de Newport News, Va., Vale Press, 1998.

JACKSON, S., "'Virtual Standpoints' and the Pragmatics of Conversational Argument", en Argumentation Illuminated, F. H. van Eemeren, R. Grootendorst, J. A. Blair y C. A. Willard (eds.), Amsterdam, Sic Sat, 1992, 260269.

JACKSON, S., "Fallacies and Heuristics", en Analysis and Evaluation. Proceedings of the Third ISSA Conference on Argumentation. Vol. II, F. H. van Eemeren, R. Grootendorst, J. A. Blair y C. A. Willard (eds.), Amsterdam, Sic Sat, 1995, 257-269.

Kock, C., "The Domain of Rhetorical Argumentation", en Proceedings of the Sixth Conference of the International Society of the Study of Argumentation, F. H. van Eemeren, J. A. Blair, C. A. Willard y B. Garssen (eds.), Amsterdam, Sic Sat, 2007, 785-788.

Mill, J. S., Utilitarianism, Liberty, Representative Government. Selections from Auguste Comte and Positivism, H.B. Acton (ed.), London, Dent, 1863/1972.

O’Keefe, D., "Pragma-Dialectics and Persuasion Effect Research", en Considering Pragma-Dialectics. A Festschrift for Frans van Eemeren on the Occasion of his 60 TH Birthday, P. Houtlosser y M.A. van Rees (eds.), Mahwah, New Jersey, Lawrence Erlbaum, 2006, 235-243.

Perelman, C. y L. Olbrechts-Tyteca, Traité de l'argumentation. La nouvelle rhétoricque, Paris, Presses Universitaires de France, 1958.

Perelman, C. y L. Olbrechts-Tyteca, Tratado de la argumentación. La nueva retórica, Madrid, Gredos, 1989.

Toulmin, S. E., The Uses of Argument, Cambridge, MA, Cambridge University Press, 1958. 